\title{
A Survey of Genetic Variation in Streptomyces Isolates Causing Potato Common Scab in the United States
}

\author{
Leslie A. Wanner
}

U.S. Department of Agriculture-Agricultural Research Services Vegetable Lab, 10300 Baltimore Ave., Beltsville, MD 20705.

Accepted for publication 10 July 2006.

\begin{abstract}
Wanner, L. A. 2006. A survey of genetic variation in Streptomyces isolates causing potato common scab in the United States. Phytopathology 96:1363-1371.

Common scab is a serious disease of potatoes and other root and tuber crops, affecting crop quality and market value. The disease is caused by gram positive soil bacteria in the genus Streptomyces. Disease incidence and severity vary in different locations and years; this is due in part to variation in the environment (weather) and genetic variation in potato cultivars. Little information is available on the contribution of genetic variation by the pathogen. To examine genetic diversity in different locations within the United States, streptomycetes were isolated from lesions on field-grown potatoes from six states. Isolates were classified into species based on sequence of variable regions in the 16s rRNA gene. The presence of genes associated with the recently described S. turgidi-
\end{abstract}

ABSTRACT scabies pathogenicity island (PAI) was also determined. About half of the isolates belonged to $S$. scabies or S. europaeiscabiei based on 16s rDNA sequence, and had characteristic features of the PAI. They were found in all six states, and were pathogenic on potato and radish. The remaining isolates included pathogens and nonpathogens. They were varied in appearance, and represent several species, including one pathogenic species not previously reported. Some pathogenic isolates lacked one or more genes characteristic of the PAI, although all had genes for biosynthesis of the pathogenicity determinant thaxtomin. In this relatively small survey, regional differences in scab-causing streptomycetes were seen. This report furnishes tools and baseline data for population genetic study of scab-causing streptomycetes in the United States.

Additional keywords: pathogenicity island markers, Solanum tuberosum, Streptomyces acidiscabies.
Common scab is a serious disease of potatoes in the United States and worldwide, reducing the quality of tubers, sometimes to the point that they can no longer be sold $(15,25)$. Common scab has been reported wherever potatoes are grown, including the Americas, India, Asia, and Africa. Scab also affects other tuber and root crops, including radish, beet, carrot, turnip, and sweet potato, and may result in considerable economic loss (15). On potato tubers, common scab symptoms range from a few superficial or raised brown spots on the skin to dark pits extending several millimeters into the potato tuber. The lesions may be small and discrete, or they may coalesce to cover large areas of the tuber surface.

Common scab is caused by gram-positive actinomycetes in the genus Streptomyces. Although the genus is diverse and abundant in the soil, only a small percentage of species cause plant disease. Plant-pathogenic streptomycetes have been classified into a number of species based on DNA sequence relatedness, 16s ribosomal DNA (rDNA) sequence, and biochemical characteristics $(1,2,5$, $14,27,33)$. The most widely described common scab pathogen is Streptomyces scabies (synonym S. scabiei, type strain ATCC 49173), which occurs worldwide. Two additional morphologically similar but genetically distinct species of streptomycetes, formerly classified as S. scabies, have been delineated by BouchekMechiche et al. (1) in France. These are S. europaeiscabiei and S. stelliscabiei; they have also been isolated in the eastern United States and Canada (5). Bukhalid et al. recognized a fourth, mor-

Corresponding author: L. A. Wanner; E-mail address: wannerl@ba.ars.usda.gov

* The $\boldsymbol{e}$-Xtra logo stands for "electronic extra" and indicates that the online version contains a figure showing the complete alignment of 16s rDNA genes from common scab-causing Streptomyces species. Figure 1 appears in color online.

DOI: 10.1094/PHYTO-96-1363

This article is in the public domain and not copyrightable. It may be freely reprinted with customary crediting of the source. The American Phytopathological Society, 2006. phologically similar group of common scab-causing streptomycetes from Egypt as similar or identical to S. bottropensis (5). Other species causing common scab include $S$. acidiscabies, described from eastern North America, Japan, and Korea (19,28, 34); S. turgidiscabies described from Japan, Korea, Finland, and Sweden $(18,21,26,28)$; S. aureofaciens from Finland (18); and three new species from Korea (27). Bouchek-Mechiche defined an additional pathogenic species, $S$. reticuliscabiei, responsible for netted scab in Europe (1). S. reticuliscabiei may be the same as or related to $S$. aureofaciens, causing russet or netted scab in Canada and in Finland $(9,18)$.

Despite the genetic diversity in pathogenic species, the genes responsible for and the mechanism(s) of pathogenicity seem to be shared among common scab-causing streptomycetes. Pathogenicity genes are clustered in a large block that has been horizontally transferred into new Streptomyces species to create pathogenic species or strains, and thus functions as a pathogenicity island (PAI) $(4,5)$. The Streptomyces PAI has been described from $S$. turgidiscabies (17). A single $425-\mathrm{kb}$ restriction endonuclease fragment contains genes for synthesis of the pathogenicity determinant thaxtomin ( $t x t A B, t x t C$, and nos) near one end, and genes for pathogenicity factors tomatinase (tomA) and a necrosis-inducing protein (necl) near the other end. These three loci are also found in a similar large contiguous chromosomal region of $S$. acidiscabies and S. scabies (17). Genetic analysis suggests that the gene clusters have been independently horizontally transferred from an $S$. scabies strain into saprophytic species to create $S$. acidiscabies and $S$. turgidiscabies $(5,13,17)$.

Severity of common scab can vary from field to field, year to year in the same field, and from cultivar to cultivar. In North America, the severity of potato common scab varies from region to region. More frequent and severe common scab is reported in the northeastern and upper Midwestern regions of the United States and the corresponding eastern and middle regions of 
Canada than is reported from the western potato-growing regions of both countries (15). Differences in common scab severity on an individual potato cultivar grown in different regions have also been reported (12).

Environmental factors contribute to common scab outbreaks; scab has been associated with dry warm seasons $(24,30)$. Maintaining high soil moisture during tuber initiation and enlargement is a widely cited scab management strategy $(20,22,24,30)$. However, scab outbreaks commonly occur in irrigated or wet soil conditions in northern Europe, Israel, and Canada $(6,10,23)$. Soil alkalinity (soil $\mathrm{pH}$ above 7 ) has traditionally been considered to favor scab, although "acid scab" occurring at a soil pH of 5.0 or below and caused by $S$. acidiscabies was first recognized in the United States in the 1950s (19). The evolution of streptomycetes well adapted to new conditions is not unexpected, considering the ubiquity of the genus combined with horizontal transfer of a PAI. Furthermore, climatic or environmental differences could result in different profiles of scab-causing streptomycetes in different regions and seasons.

There has been much speculation on whether the different groups or species of Streptomyces produce differences in scab symptoms, and streptomycetes specifically responsible for pitted, raised, netted, or other types of scab have been described $(1,8,10,18)$. When it was originally described, $S$. turgidiscabies was reported to cause severe corky lesions, in contrast to pitted or other lesion types caused by $S$. scabies (26), but this difference has not been upheld in more recent assessments of scab symptoms caused by $S$. turgidiscabies $(16,18)$. French researchers have recently reported that some of the European scab-causing species do induce different symptom profiles, and they also differentially affect different potato cultivars (29). In particular, S. reticuliscabiei causes netted scab $(3,29)$, which affects all underground parts of the plant, and affects only a few cultivars. Specific groups of the scab pathogen also appear to be responsible for russet scab in North America (9). Adding to the complexity of scab disease development, Finnish researchers have demonstrated that multiple scabcausing species can be isolated from the same field, potato cultivar, tuber, and even from a single lesion (21). Although it is clear that the severity of scab symptoms is related to the susceptibility of potato cultivars, it seems possible that different populations or profiles of scab pathogens could be responsible for some of the differences in potato common scab frequency and severity reported in different regions in North America or elsewhere.

In order to understand the importance of different species or populations of pathogenic streptomycetes in the development of common scab in the field, it is necessary to have an initial idea of the total diversity in plant-pathogenic Streptomyces inhabiting different locations. No systematic effort to catalog the different types (and abundances) of scab-causing strains in the United States has been reported. One objective of this study was to develop an initial picture of phylogenetic groups represented in plant-pathogenic Streptomyces isolates obtained from common scab lesions on potatoes grown in different regions of the United States. To do this, species-specific polymerase chain reaction (PCR) primers were developed to unique regions of the 16s rDNA gene in different Streptomyces species. Additional Streptomyces species-specific primers similar to those used to identify scab-causing streptomycetes in Scandinavia (21) were also employed. A second objective was to investigate genetic diversity in the PAI of these isolates. To accomplish this objective, the presence of three genes/operons encoding pathogenicity determinants or pathogenicity factors, approximately demarking the "ends" of the PAI in S. turgidiscabies (17), was assessed.

\section{MATERIALS AND METHODS}

Streptomyces isolation and morphological characterization. Ninety-nine streptomycetes isolated from field-grown scabby potatoes were compared in this study. Forty-eight of these isolates were previously partially characterized (35). Streptomycetes were isolated from scabby potato tubers obtained from collaborators in six U.S. states. The procedure for isolating streptomycetes from scabby potato tubers was described previously (35). Potatoes from Maine came from the same scab test plot in three successive years. New York isolates came from one location in 1 year. Ohio isolates came from one location in two different years, and Michigan isolates came from two locations in the same year. Wisconsin isolates came from four widely separate locations in two different years, and Idaho isolates came from at least four separate locations in three successive years. Isolations were made from common scab lesions on numerous potato varieties, and from various types of lesions, ranging from superficial corky lesions to deep coalescing pitted lesions. The first two letters in an isolate's name designate what state it came from, and the second two digits designate the year in which scabby tubers were harvested.

Culture and storage of isolates. Yeast-malt extract (YME) or YME-agar (32) was used for routine culture of Streptomyces strains. Morphological characteristics noted included the color of the substrate mycelium, the color of spores, and the color of diffusible pigment produced (if any) on YME. Production of dark diffusible melanoid pigment on peptone-yeast extract iron (PYI) agar was also noted. Strains were maintained as spore suspensions in $20 \%$ glycerol at $-80^{\circ} \mathrm{C}$ and/or as agar plugs cut from actively growing plates and stored at $-80^{\circ} \mathrm{C}$.

Pathogenicity testing. To assay pathogenicity, radishes and/or potatoes were planted into a soil/sand mixture infested with a single Streptomyces isolate at a known density. Streptomyces cultures to be mixed with soil were prepared as described (35) with minor modifications. Briefly, $50 \mathrm{ml}$ of YME in a $250-\mathrm{ml}$ Erlenmeyer flask was inoculated with $4 \times 10^{6}$ Streptomyces spores harvested in water from a 2- to 3-week-old YME plate. Cultures were grown for 3 days, with shaking, at $28^{\circ} \mathrm{C}$. Cells were pelleted by centrifugation and resuspended in one-third the original volume of sterile distilled water. Five milliliters of this resuspended 3 -day culture and $50 \mathrm{ml}$ of sterile $2 \times$ Say solution (40 g of sucrose, $2.4 \mathrm{~g}$ of asparagine, $1.2 \mathrm{~g}$ of $\mathrm{K}_{2} \mathrm{HPO}_{4}$, and $20 \mathrm{~g}$ of yeast extract in 1 liter of sterile distilled water [8]) were added to a bag containing $300 \mathrm{~cm}^{3}$ of sterile vermiculite. Inoculated vermiculite was incubated for 14 days at $28^{\circ} \mathrm{C}$, and was mixed in the bags every other day during incubation. After 14 days, an approximately $1-\mathrm{cm}^{3}$ sample of the vermiculite culture was shaken in $10 \mathrm{ml}$ of sterile distilled water. Ten microliters of dilutions $\left(10^{-2}\right.$, $10^{-3}, 10^{-4}, 10^{-5}$ ) of this extract was plated in quadruplicate onto YME agar. Plates were incubated at $28^{\circ} \mathrm{C}$ for 2 days, and colonies were counted to ensure that only Streptomyces was visible in the inoculum, and to estimate inoculum concentrations.

For both potato and radish pathogenicity testing, $15-\mathrm{cm}$ pots were partly filled with $600 \mathrm{~cm}^{3}$ of a 50:50 mixture of sterile sand/ potting soil (Jiffy Mix Plus, Jiffy Products of America, Batavia, IL). Three hundred cubic centimeters of vermiculite inoculum, usually containing 1 to $10 \times 10^{10} \mathrm{CFU}$ Streptomyces, was added to $2,700 \mathrm{~cm}^{3}$ of $50: 50$ sterile sand/soil and mixed thoroughly. Twelve hundred cubic centimeters of inoculum-containing mixture was overlaid in each pot $\left(\geq 4 \times 10^{9} \mathrm{CFU}\right.$ per pot; $1.3 \times 10^{6}$ CFU per $\mathrm{cm}^{3}$ of soil). Potato tubers were surface-disinfected for $5 \mathrm{~min}$ in $0.5 \%$ bleach $/ 0.5 \%$ soap to eliminate other strains of Streptomyces that could be present on the tuber, and rinsed in sterile distilled water before seed pieces were planted directly in the inoculum-containing layer. Potato cultivars used were Superior (moderately resistant to common scab) and Green Mountain (susceptible to common scab) (11). Certified seed potatoes were obtained from Maine Farmers Exchange (Presque Isle, ME), Jewel Brothers (Monticello, ME) or Cleaves Farms (Sangerville, ME). Disinfected radish seeds, cv. Cherriette F1 (Johnny's Selected Seeds, Albion, ME), were planted 9 to 10 per pot, as described (35). 
Potatoes and radishes were grown in Conviron growth chambers (Controlled Environments Ltd., Winnipeg, Manitoba, Can.) at $24^{\circ} \mathrm{C}$ with a $14-\mathrm{h}$ photoperiod. Light (200 to $250 \mu \mathrm{mol} \mathrm{m} \mathrm{m}^{2}$ ) was provided by Philips natural daylight fluorescent lamps (Philips Lighting Co., Somerset, NJ). Soil was permitted to dry between waterings. Potatoes were harvested and scored for scab after 16 weeks, and radishes were harvested after 4.5 weeks. Three replicate pots per isolate (per plant cultivar) were included in each experiment. Ninety-two isolates were assayed for pathogenicity in radish, 60 of the isolates in two or more independent experiments. Thirty-four isolates were assayed for pathogenicity in potato, 19 of them in two or more independent experiments.

Scab disease index. Scab lesions were rated on all potato tubers larger than $0.5 \mathrm{~cm}$ in diameter after 4 months of growth. Potatoes were scored for severity of scab lesions and percent area covered by lesions. The lesion severity index scale used was as follows: $0=$ no lesions; 1 = superficial lesions, $<10 \mathrm{~mm}$ in diameter; 2 = superficial lesions, $>10 \mathrm{~mm}$ in diameter; $3=$ raised lesions, $<10 \mathrm{~mm}$ in diameter; 4 = raised lesions, $>10 \mathrm{~mm}$ in diameter; and $5=$ pitted lesions. The final disease index was assigned on a scale of 0 to 500 , which was calculated by multiplying the average lesion severity of all scorable tubers in a pot by the average percent area covered by lesions. Isolates producing ratings of $\geq 20$ were scored as pathogenic. Rating of scab lesions on radishes on a 0 to 5 scale was as described (35). Isolates producing ratings of $\geq 1.0$ were scored as pathogenic.

DNA isolation. For DNA isolation, Streptomyces cultures were grown at $28^{\circ} \mathrm{C}$ for 3 days in YME, and cells were pelleted to obtain approximately $100-\mathrm{mg}$ aliquots that were stored frozen at $-20^{\circ} \mathrm{C}$ until use. Alternatively, plugs were taken from 2- to 3-week-old YME plates of a Streptomyces isolate using a sterile no. 2 cork borer. DNA was isolated from frozen cells or from two to four agar plugs with a Bio101 FAST DNA kit (sphere plus garnet beads and CLS-Y lysing solution) and a Qbiogene Fast Prep machine (settings: speed, 5.0; time, $25 \mathrm{~s}$; both machines: MP Biomedicals United States, Solon, $\mathrm{OH})$ ). DNA was eluted from columns in $2 \times 50 \mu \mathrm{l}$ of $10 \mathrm{mM}$ Tris- $\mathrm{HCl}(\mathrm{pH} \mathrm{9.0)}$ and $0.2 \mathrm{mM}$ EDTA. Suitability of DNA preparations for PCR amplification was verified by PCR amplification of $16 \mathrm{~S}$ rRNA genes using the primer pairs $16 \mathrm{~s} 1 \mathrm{~F}$ and $16 \mathrm{~s} 455-435$ or $16 \mathrm{~s} 1 \mathrm{~F}$ and $16 \mathrm{~s} 1 \mathrm{R}$ (Table 1).

PCR analysis of marker genes in the PAI and the 16s rRNA gene. Analysis of the presence of genes characteristic of the $S$. turgidiscabies PAI, as described in Kers et al. (17), was carried out by PCR, using primers specific to the $t x t A B$ operon (this study), the tomA gene (this study), and the necl gene (4). Primers for detection of the $t x t A B$ and tom $A$ genes were designed based on sequence data available in GenBank ( $S$. acidiscabies thaxtomin synthetase A [txtA] and thaxtomin synthetase B [txtB] operon, accession no. F255732; S. turgidiscabies tomatinase tomA gene, accession no. AY707079). Three primer pairs were designed for each locus, and the pair giving the most sensitive and specific PCR amplification was chosen for use in this study. Primer pairs used, expected PCR product sizes, and details of PCR conditions are listed in Table 1.

Isolates were assigned to Streptomyces species based on the results of PCR with a series of primer pairs designed to specifically amplify 16s rRNA genes from different species (Table 1; Fig. 1). To design these primers, 16s rDNA sequences for the following species were obtained from GenBank and aligned using Clustal X: S. scabies type strain ATCC 49173 (gi971124) (1,5), S. europaeiscabiei (gi5263068 and AB026208) (1), S. stelliscabiei (gi5263074 and AB026212) (1), S. bottropensis (AB026215 and gi971133), S. acidiscabies ${ }^{\mathrm{T}}{ }^{\left({ }^{\mathrm{T}}\right.}$ indicates type strain) ATCC 49003 (AB026220), S. turgidiscabies ${ }^{\mathrm{T}}$ ATCC 700249 (AB026221), S. aureofaciens (gi3550677), and S. diastatochromogenes (gi971132). Most of the approximately 1,500-bp sequence is identical in all of these species, but three regions of variability were readily apparent (Fig. 1), as described by Kreuze et al. (18) and Bukhalid et al. (5). PCR primers were designed to the variable regions with the help of the program Primer Premiere version 5.0 (Premiere Biosoft International, Palo Alto, CA). Locations and sequences of the species-specific primers are shown in Figure 1, aligned with the variable sequence regions of the Streptomyces species. Specific amplification of $16 \mathrm{~s}$ rDNA gene sequences was verified using DNA prepared from a panel of Streptomyces isolates and strains, including Streptomyces sp. ATCC 10246 (albidoflavus cluster; negative control), S. scabies ${ }^{\mathrm{T}}$ ATCC 49173 (S. scabies), S. acidiscabies ${ }^{\text {T }}$ ATCC 49003, S. scabiei ATCC 33282 (S. europaeiscabiei), S. scabiei ATCC 33281, S. turgidiscabies $^{\text {T }}$ ATCC 700248, Streptomyces sp. ATCC 700525, S. aureofaciens ATCC 700526, Streptomyces sp. ATCC 700528, and isolates ID01-12c, NY02-3A, and ID03-2B. DNA from all of these Streptomyces species/strains was used as a PCR template with seven species-specific primer pairs to confirm specificity. Primer pair scab1m/scab2m or primer pair ASE3/scab2m was used to identify S. scabies and S. europaeiscabiei, which differ by three separated nucleotides and could not be distinguished by PCR in this study. One of these nucleotide differences is located in primer scab1m versus primer scab1 (Fig. 1).

Primers were purchased from Integrated DNA Technologies (Coralville, IA). PCR analyses were carried out in $50 \mu \mathrm{l}$ containing $10 \mathrm{mM}$ Tris- $\mathrm{HCl}(\mathrm{pH} 9.0), 50 \mathrm{mM} \mathrm{KCl}, 0.1 \%$ Triton $\mathrm{X}$ $100,1.0$ to $2.0 \mathrm{mM} \mathrm{MgCl}, 200 \mu \mathrm{M}$ each dNTP, 25 pmol each primer, $2.5 \mathrm{U}$ of Taq DNA polymerase (Promega Corp., Madison, WI), 2 to $25 \mathrm{ng}$ of DNA template (1 $\mu$ l of a 1:9 dilution), and MilliQ water. Amplification was carried out in a Perkin-Elmer Thermal Cycler 480 (PerkinElmer Inc., Wellesley, MA) programmed for one initial denaturation step at $95^{\circ} \mathrm{C}$ for $3 \mathrm{~min}$, followed by 40 cycles of denaturing at $95^{\circ} \mathrm{C}$ for $20 \mathrm{~s}$, annealing for $30 \mathrm{~s}$ and extension at $72^{\circ} \mathrm{C}$ for $2 \mathrm{~min}$, and ending with a $4^{\circ} \mathrm{C}$ hold. Annealing temperature and concentration of $\mathrm{MgCl}_{2}$ were optimized to yield specific amplification for each primer pair; these conditions are specified in Table 1, together with sequences of PCR primers, expected product sizes, and the DNA template used as a positive control. Suitability of all DNA templates for PCR was first verified using primer pair $16 \mathrm{~s} 1 \mathrm{~F}$ and $16 \mathrm{~s} 455-435$ or $16 \mathrm{~s} 1 \mathrm{~F}$ and 16s1R (Table 1). Amplified DNA fragments were separated on $1.5 \%$ agarose gels and stained with ethidium bromide. PCR amplification experiments were repeated at least twice with at least two independently cultured and isolated DNA template preparations for each Streptomyces isolate. All three pairs of primers to genes in the PAI, and all seven primer pairs to specific $16 s$ rDNA species or groups were used on all templates in this study.

Cloning and sequencing of 16s rRNA genes. The 16s rRNA gene was amplified from DNA of 15 Streptomyces isolates by PCR using primers $16 \mathrm{~s} 1 \mathrm{~F}$ and $16 \mathrm{~s} 1 \mathrm{R}$ (5). The 1,500-bp PCR product was excised and eluted from an agarose gel using a Zymoclean Gel DNA Recovery Kit (Zymo Research Corp., Orange, CA). DNA was cloned into pGEM-T Easy (Promega Corporation). Six white colonies were picked for each gene and DNA was prepared by an alkaline lysis quick prep procedure (31). Clones verified to contain the correct-sized insert were sequenced or partially sequenced using the dideoxy-chain termination method and dye terminator labeling on an ABI Prism 310 Gene Analyzer at the U.S. Department of Agriculture Agricultural Research Services Floral and Nursery Crops Laboratory (Beltsville, MD) or an ABI 4300 by Macrogene, Inc. (Seoul, South Korea). Primers used are listed in Table 1, and included some or all of the following: T7 promoter primer, SP6 promoter primer, 16s-1F, 16s 455-435, 16s-3F, 16s-2R, 16s 873-893, 16s 1366-1346, 16s 1346-1366, and 16s-1R. Sequences were assembled using the Seqman subprogram of DNA Star, version 4.05 (DNASTAR Inc., Madison, WI). Partial nucleotide sequences were obtained for the 16s rRNA genes of isolates ME01-11h, ME01-17h, ME01-18h, MI02-2, NY02-1A, NY02-1BNY02-3C, 
OH01-5A, OH01-6.2A, OH01-12c, and WI02-5. Complete nucleotide sequence was obtained for the 16s rRNA gene of isolates ID01-12c, ID01-6.2a, NY02-3A, and OH01-5D, and nearly complete sequence was obtained for ID01-16c. Sequences of variable regions are shown in Figure 1, and complete sequences have been deposited as GenBank accession nos. DQ341443 (ID0112c), DQ861639 (ID01-6.2A), DQ861640 (NY02-3A), DQ861637 (OH01-5D), and DQ861638 (ID01-16C).

\section{RESULTS}

In this small survey, pathogenicity on potato and radish, phylogenetic affiliation based on 16s rDNA sequence, and characteristics of the recently described $S$. turgidiscabies PAI were assessed in streptomycetes isolated from field-grown potatoes from different regions of the United States. Differences in gene content within the PAI and differences in sequence of the variable regions of the 16s rRNA gene were identified.

Morphological characteristics. Isolates were variable in their appearance and in production of pigments on YME and PYI medium (data not shown). Many isolates were morphologically similar to the $S$. scabies type strain, ATCC 49173; on YME agar they produced a tan to caramel-brown substrate mycelium, gray spores, and a brownish orange diffusible pigment. Pathogenic isolates had substrate mycelium colors ranging from cream and clear light gold to tan and caramel-brown, and produced spores ranging in color from white and light gray to dark gray or nearly black. Colors of the substrate mycelium and of diffusible pigments produced on YME were more varied among the nonpathogenic isolates. Two-thirds of the isolates produced inky black melanoid pigments on PYI, including both pathogens and nonpathogens.

TABLE 1. Primer pairs and conditions used in polymerase chain reaction (PCR) detection of genes in the Streptomyces pathogenicity island (PAI) and speciesspecific primers to the $16 \mathrm{~s}$ rRNA gene

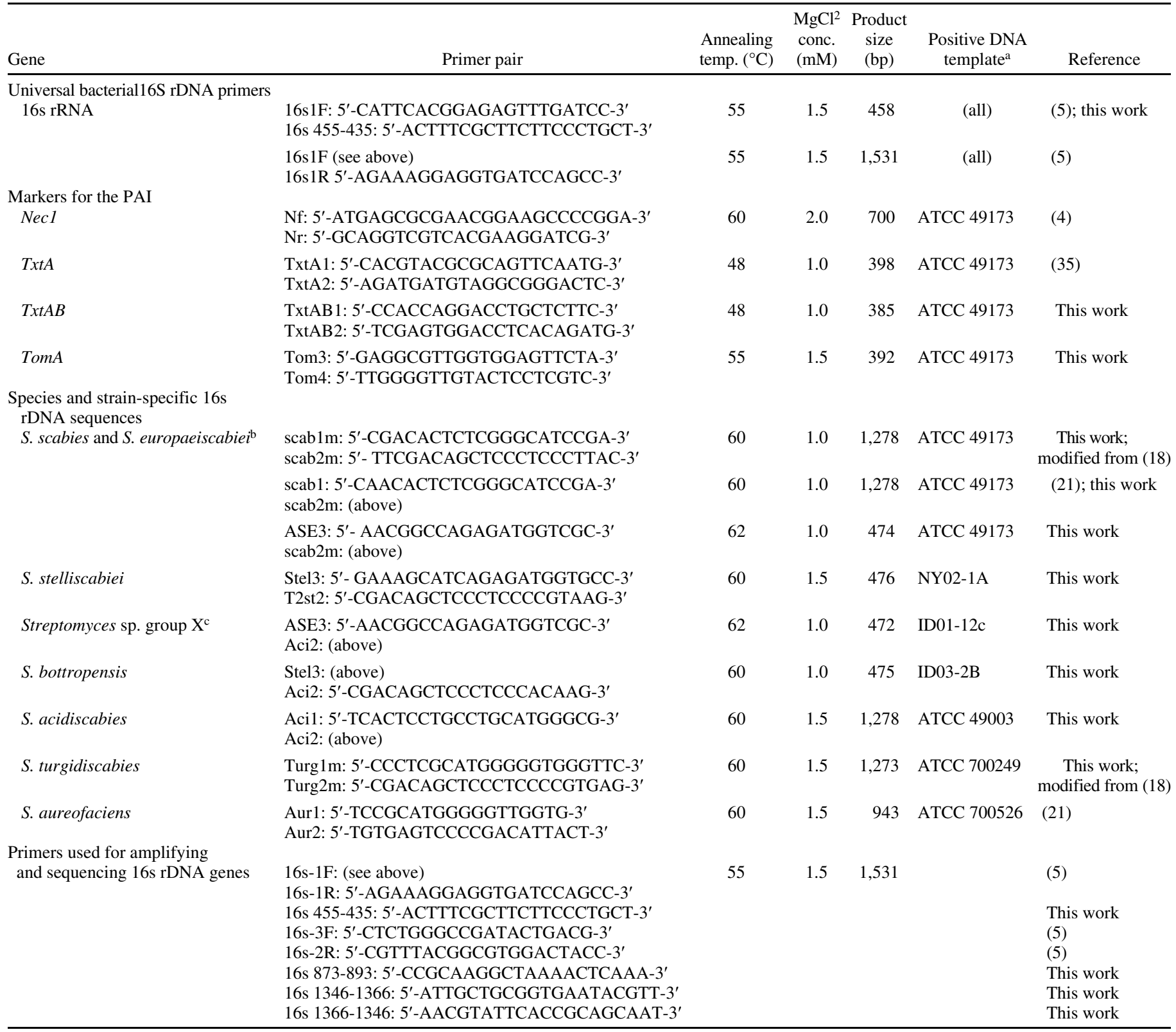

a ATCC (American Type Culture Collection) number, or isolate number from this study.

${ }^{\mathrm{b}}$ PCR under these conditions with primer pair scab1/scab2m and primer pair scab1m/scab2m do not differentiate $S$. scabies and $S$. europaeiscabiei although the primer scab1m sequence is perfectly homologous to $S$. scabies and has $1 \mathrm{bp}$ mismatch to $S$. europaeiscabiei, and primer scab1 is perfectly homologous to S. europaeiscabiei and has 1 bp mismatch to $S$. scabies.

c Newly identified group of scab-causing Streptomyces. 
Pathogenicity. Presence or absence of the $t x t A B$ genes, encoding the synthetase for the phytotoxic pathogenicity determinant thaxtomin, was assessed by PCR in all 99 isolates. Fifty-nine were $\operatorname{txt} A B+$, and therefore presumed to be pathogenic, and 40 were not. The correlation between pathogenicity and $\operatorname{txt} A B$ was confirmed in nearly all of the isolates by planting potatoes or radishes in soil infested with the isolate at a measured inoculum density. Ninety-two isolates were tested for pathogenicity in radish. Of these, 57 of 59 isolates positive for $t x t A B$ by PCR were judged to be pathogens, having a disease index of $\geq 1.0$ on a 0 to 5 scale (summarized in Table 2), and two txtAB-positive isolates were not tested. Twenty-four positive and 10 isolates negative for txt $A B$ by PCR were tested for pathogenicity in one or more varieties of potato. Again, all $t x t A B$-positive isolates were pathogenic, and no $t x t A B$-negative isolate was pathogenic. Results of pathogenicity testing were usually unambiguous, and the results of testing in radish corresponded to results in potato. In the few cases where pathogenicity testing gave ambiguous results, an explanation was usually apparent. In several cases of mixed results from multiple independent pathogenicity tests of an isolate in radish and/or potato, scab indices below the threshold for pathogenicity of 1.0 in radish or 20 in potato were associated with low inoculum densities used in the initial infestation of pots. This observation underscores the necessity for information on the quantity of pathogen going into the assay.

Molecular characterization of isolates. Three regions of sequence variability in the 16s rRNA gene have been identified that are useful in delineating the species of common scab-causing streptomycetes $(5,18,21)$. To investigate the diversity in Streptomyces species in the U.S. isolate collection, PCR primers were used to specifically amplify $S$. scabies/S. europaeiscabiei, S. stelliscabiei, S. acidiscabies, S. bottropensis, S. turgidiscabies, and $S$. aureofaciens. Finnish researchers previously developed primer pairs specific to $S$. aureofaciens and $S$. turgidiscabies, as well as a primer pair that amplified a product from S. scabies ATCC 49173 (21). Their primer pair (scabI and scabII) was modified in the present study (to scab1m and scab2m) so that the primer to the $\gamma$ variable region was perfectly homologous to the $S$. scabies $16 \mathrm{~s}$ rDNA sequence, and the primer homologous to the 1435 variable region contained a single nucleotide present in sequence data available for $S$. scabies and $S$. europaeiscabiei, but missing in the original scabII primer (21). Additional primer pairs were developed in this study based on differences in 16s rDNA variable regions that permitted all pathogenic isolates in this collection to be assigned to a Streptomyces species (Fig. 1, Table 2).

Seven Streptomyces species-specific primer pairs and three PAI marker gene primer pairs were used to characterize the U.S. isolate collection (Table 1). The largest number of isolates, 49 isolates, belonged to $S$. scabiei or S. europaeiscabiei, based on rDNA sequence (Table 2). These were found in all six states, and were the only or the predominant type in Ohio, Michigan, Wisconsin, and Maine. Nearly all (47 isolates) of these isolates, were $t x t A B$ positive, and 44 had all three PAI marker genes. All 46 txtABpositive isolates tested were pathogenic on radish. Two isolates with the $S$. scabiei/S. europaeiscabiei 16s rDNA type, one from Michigan and one from Idaho, had $\operatorname{txt} A B$ but were missing both necl and tomA. These isolates were previously identified by both PCR and Southern blotting as missing the necl gene and were pathogenic in both radishes and potatoes (35).

Another isolate lacked the necl gene but had tomA and was pathogenic on radish and potato. One isolate with the $S$. scabieil $S$. europaeiscabiei 16s rDNA type lacked all three PAI genes and is presumably nonpathogenic, and another lacking $\operatorname{txt} A B$ but having necl and tomA was not pathogenic. S. scabies and S. europaeiscabiei, which differ by a single nucleotide in the regions covered by species-specific primers (and only three nucleotides in the entire 16s rDNA gene), were not separated with the scab1m/ $\mathrm{scab} 2 \mathrm{~m}$ primer set used in this study. To determine whether both
S. scabies and S. europaeiscabiei were represented in the U.S. collection, the 16s rRNA gene was cloned and sequenced from several isolates. Complete or partial 16s rDNA sequence was obtained for nine pathogenic and one nonpathogenic S. scabies or $S$. europaeiscabiei isolate. These two species have the same sequence in the 16s rDNA 1435 and alpha variable regions, but are distinguished by $\mathrm{G}$ or $\mathrm{A}$ at position 172 in the gamma variable region (nucleotides 160 to 200; Fig. 1 [5]). Seven isolates belonged to S. scabies (four from Ohio, one from New York, and two from Maine), and three belonged to S. europaeiscabiei (one each from Wisconsin, Michigan, and Idaho). Although this is too few isolates on which to base strong conclusions, it appears that $S$. scabies is more common in the eastern United States, and S. europaeiscabiei in the west.

Seven isolates were classified as S. stelliscabiei (S. scabies species complex group 3 as defined by Bukhalid et al. [5]) using the species-specific PCR primers (Table 2). All were pathogenic and had all three PAI marker genes. Six of these isolates came from a single location in New York; the seventh came from Idaho. S. stelliscabiei has also been found in France and South Africa $(1,5)$.

No isolate was found that unambiguously belonged to $S$. bottropensis, previously described as a strain in the $S$. scabies complex from Egypt (5). Two isolates produced a strong PCR product with a $S$. bottropensis-specific primer pair, but also consistently produced a weak product with the $S$. stelliscabiei-specific primers. These isolates were not pathogenic, and no PCR products were obtained with the three primer pairs to genes characteristic of the PAI.

Five isolates from Idaho were identified that were pathogenic on radish, but for which none of the 16s rDNA species-specific primer pairs yielded a product. Two of these isolates were tested and found to be pathogenic in potato as well as radish (35). The 16s rDNA region was cloned and sequenced from two isolates of this type (L. Wanner, unpublished data). The sequences were identical to each other and closely related to S. scabies, S. europaeiscabiei, and $S$. bottropensis. The sequence of two out of the three variable regions in the $16 \mathrm{~s}$ rRNA gene is identical to that of $S$. europaeiscabiei, but the sequence differs in the third variable region centered around nucleotide 1435. In this region, the sequence is identical to a pathogenic $S$. bottropensis, as well as to (pathogenic) $S$. acidiscabies and to nonpathogenic $S$. diastatochromogenes and $S$. bottropensis. A PCR primer pair designed to amplify a product from this combination of variable $16 \mathrm{~s}$ rDNA sequences amplified a product from a total of six Idaho isolates (Fig. 1, Table 2). Five of these were pathogenic and contained the txtAB operon, and one lacked this operon and was not pathogenic. The five pathogenic Idaho isolates were characterized by having the $t x t A B$ and tom $A$ genes, but lacking the necl gene as determined by PCR; absence of a close homolog to the necl gene was confirmed in two of these isolates by Southern blotting (35). These isolates comprise a fifth group of species morphologically similar but genetically distinct from species previously described in the $S$. scabies complex. This new member of the S. scabies complex is referred to as group $\mathrm{X}$ in Figure 1 and in Tables 1 and 2. Two additional nonpathogenic isolates with this $16 \mathrm{~s}$ rDNA type were identified from Wisconsin; all three lacked the three PAI markers (L. Wanner, unpublished data). All 59 pathogenic U.S. isolates could be classified into one of the four Streptomyces species or species groups: S. scabies/S. europaeiscabiei, S. stelliscabiei, or the new group X. No example of $S$. turgidiscabies or $S$. aureofaciens was found in this isolate collection.

Among nonpathogenic isolates, only 10 could be readily classified into one of the common scab-causing Streptomyces species based on 16 s rDNA sequence. Two fell into $S$. scabies or $S$. europaeiscabiei, three into group $\mathrm{X}$, and three could be tentatively classified as $S$. bottropensis as discussed above. In addition, two isolates were identified as $S$. acidiscabies, one from Maine, and one from Wisconsin. Both were nonpathogenic, and they ap- 
peared to differ from each other in the $\alpha$ variable region of the 16s rDNA sequence. A PCR product of the expected 473-bp size was obtained for ME02-6987A with the primer pair ASE3 and acidi2, which recognizes the $S$. acidiscabies ATCC $49003^{\mathrm{T}} 16 \mathrm{~s}$
rDNA sequence, but this primer pair did not amplify a fragment from WI02-9. However, the expected 1,278-bp PCR product was obtained from both of these isolates as well as from the $S$. acidiscabies type strain with primer pair aci1/aci2.

\section{Y variable region}

scablm
971124 scabiei
scabI
AJ007423 europaeiscabiei
D63868 bottropensis
ID01-6.2A group X
Stel1
5263074 stelliscabiei
acidi1
AB026220
turg1m
AB026221
971132 turgidiscabies

OHO1-5A

OH01-5D

OH01-6.2A

OH01-12C

NY02-1B

ME01-11h

ME01-17h

ME01-18h

MI02-2

WI02-5

ID01-16C

ID01-6.2A

ID01-12C

NY02-1A

NY02-3A

NYO2 $-3 \mathrm{C}$

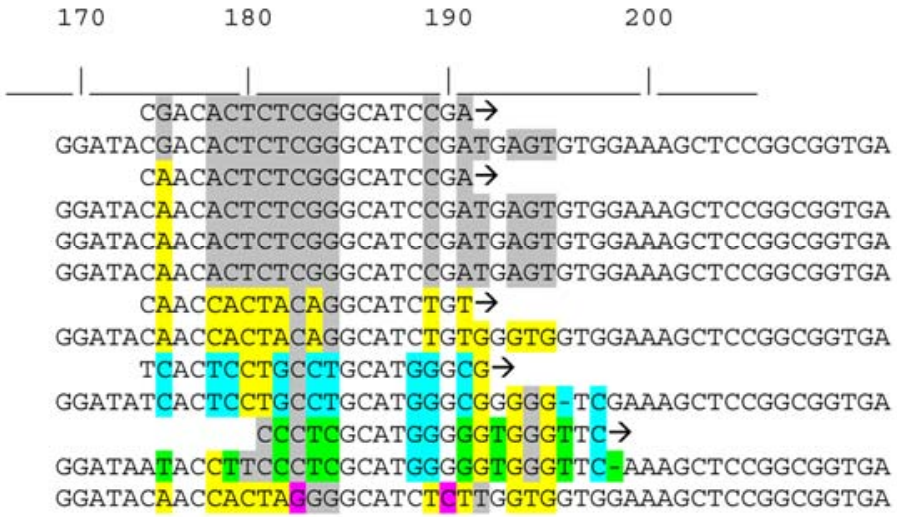

GGATACGACACTCTCGGGCATCCGATGAGTGTGGAAAGTCCGGCGGTA GGATACGACACTCTCGGGCATCCGATGAGTGTGGAAAGCTCCGGCGGTGA GGATACGACACTCTCGGGCATCCGATGAGTGTGGAAAGCTCCGGCGGTGA GGATACGACACTCTCGGGCATCCGATGAGTGTGGAAAGCTCCGGCGGTGA GGATACGACACTCTCGGGCATCCGATGAGTGTGGAAAGCTCCGGCGGTGA GGATACGACACTCTCGGGCATCCGATGAGTGTGGAAAGCTCCGGCGGTGA GGATACGACACTCTCGGGCATCCGATGAGTGTGGAAAGCTCCGGCGGTGA GGATACGACACTCTCGGGCATCCGATGAGTGTGGAAAGCTCCGGCGGTGA GGATACAACACTCTCGGGCATCCGATGAGTGTGGAGAGCTCCGGCGGTGA GGATACAACACTCTCGGGCATCCGATGAGTGTGGANAGCTCCGGCGGTGA GGATACAACACTCTCGGGCATCCGATGAGTGTGGAAAGCTCCGGCGGTGA GGATACAACACTCTCGGGCATCCGATGAGTGTGGAAAGCTCCGGCGGTGA GGATACAACACTCTCGGGCATCCGATGAGTGTGGAAAGCTCCGGCGGTGA GGATACAACCACTACAGGCATCTGTGGGTGGTGGAAAGCTCCGGCGGTGA GGATACAACCACTACAGGCATCTGTGGGTGGTGGAAAGCTCCGGCNGTGA GGATACAACCACTACAGGCATCTGTGGGTGGTGGAAAGCTCCGGCGGTGA

\section{$\alpha$ variable region}

\begin{tabular}{|c|c|}
\hline ASE3 & \\
\hline $971124 \mathrm{sca}$ & Eabiei \\
\hline AJ007423 & europaeiscabiei \\
\hline 971132 & diastatochromog \\
\hline $\mathrm{AB} 026220$ & acidiscabies \\
\hline ID01-6.2A & A group $x$ \\
\hline Stel3 & \\
\hline 5263074 & stelliscabiei \\
\hline D63868 & bottropensis \\
\hline Turgret 3 & \\
\hline $\mathrm{AB} 026221$ & turgidiscabies \\
\hline & OH01-5D \\
\hline & OH01-6.2A \\
\hline & NY02-1B \\
\hline & ME01-11h \\
\hline & ME01-17h \\
\hline & MI02-2 \\
\hline & ID01-16C \\
\hline & ID01-6.2A \\
\hline & ID01-12C \\
\hline & $\mathrm{NY} 02-3 \mathrm{~A}$ \\
\hline
\end{tabular}

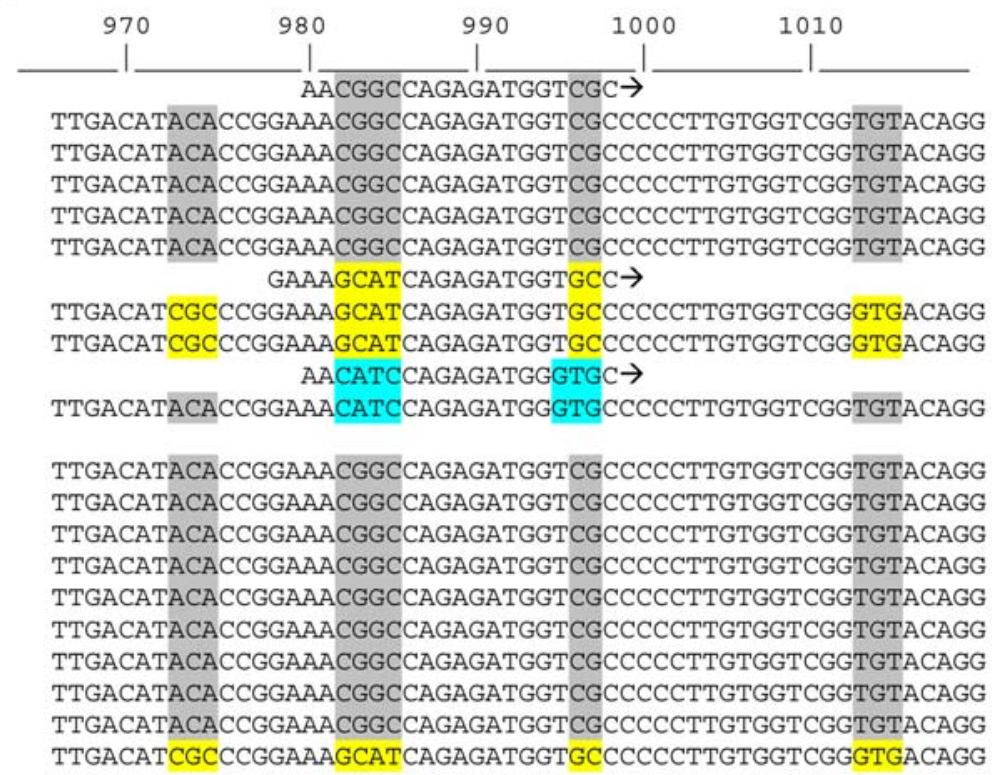

(continued on next page)

Fig. 1. Variable sequence regions in the 16s rDNA of pathogenic Streptomyces species and groups and the nonpathogenic S. diastatochromogenes. Nucleotide numbering is the same as used in Bukhalid et al. (5), and is based on the S. ambifaciens 16s rDNA sequence. Sequence information was obtained from GenBank; accession numbers precede the Streptomyces species designations to the left of each sequence region. Sequence-specific primers are indicated in boldface type, and primer sequences are integrated into the sequence alignments. Sequences from isolates in the U.S. collection are presented under each of the Streptomyces species alignments. 
Strong PCR products characteristic of both $S$. stelliscabiei and $S$. bottropensis were routinely obtained from nearly half of the 40 nonpathogenic isolates (Table 2). The explanation for this is not known; 16s rDNA genes would need to be cloned and sequenced from these isolates to find out. No product was obtained with any of the seven sets of species-specific 16s rDNA PCR primers for an additional 13 isolates; these cannot be classified on the basis of rDNA sequence without further study. Most nonpathogens lacked all three genes characteristic of the Streptomyces PAI, but two isolates had some of these (Table 2). An isolate from Maine had a tomA gene, but lacked necl and txtAB. A Wisconsin isolate had both necl and tomA, but lacked $t x t A B$.

\section{DISCUSSION}

At least four common scab-causing Streptomyces species have been previously described from North America. Three are morphologically similar to $S$. scabies: These are $S$. scabies, S. europaeiscabiei, and $S$. stelliscabiei $(1,5,14)$; the fourth is $S$. acidiscabies (19). In the present study of streptomycetes from six states in the United States, four common scab-causing species were found: S. scabies, S. europaeiscabiei, S. stelliscabiei, and the new group $X$. The largest number of common scab-causing U.S. isolates belonged to S. scabies or S. europaeiscabiei based on $16 \mathrm{~s}$ rDNA sequence. These species seem to be the prevalent scab-causing streptomycetes worldwide and have been described in South Africa, Canada, Japan, Korea, Finland, and Sweden $(1,5,21,33)$. Most isolates classified as $S$. scabies or $S$. europaeiscabie $i$ were pathogenic although a few isolates in both Scandinavia and the United States were not, including two (out of 49) in this study. Most S. scabies/S. europaeiscabiei isolates in this study had all three PAI marker genes, including $t x t A B$ and those encoding pathogenicity factors necl and tomatinase. All pathogenic isolates from Ohio, Wisconsin, and Maine, most from Michigan, and nearly half of the Idaho isolates were of this type.

The number of isolates examined from three of the six states was small, but there appears to be regional variation in the prevalence of Streptomyces species (Table 2). S. scabies/S. europaeiscabiei were the only pathogenic species identified in Wisconsin,
Maine, Michigan, and Ohio. Pathogenic S. scabies or S. europaeiscabiei were also found in Idaho and New York, but were not the predominant species. Most New York isolates belonged to S. stelliscabiei based on 16s rDNA sequence, and had all three PAI marker genes. Several species or groups were found in Idaho. Larger collections of isolates from different regions will be required to establish whether the differences in prevalence of Streptomyces species in different regions of the United States seen here are typical.

Using the species-specific PCR primers described here, most of the pathogenic isolates studied could be assigned to $16 \mathrm{~s}$ rDNA groups previously identified in scab-causing streptomycetes. However, no PCR product was found for several pathogenic isolates from Idaho. Sequencing of the 16s rRNA gene from two of these isolates showed that they had a unique combination of variable sequence regions. A substantial fraction of the isolates from Idaho belonged to this newly discovered group characterized by a unique rDNA sequence and two of the three PAI marker genes. Isolates of this type were found in two different years in Idaho (L. Wanner, unpublished data).

Comparison of the present study with the results of two small surveys in Europe $(1,18)$ and one in Korea $(28)$ suggests that there may be differences in the population profiles of Streptomyces species causing common scab in different parts of the world. In a study of 35 pathogenic and nonpathogenic streptomycetes isolated from scab lesions on potatoes in Sweden and Finland, three species were identified as responsible for potato scab $(18,21,23)$. Scandinavian scab-causing species included S. scabies (and/or S. europaeiscabiei), S. turgidiscabies, and S. aureofaciens (18); neither of the latter species was identified in the U.S. collection. Among 18 pathogenic isolates from France, 13 were $S$. europaeiscabiei, four were $S$. stelliscabiei, and only one was S. scabies (1). Of 10 U.S. isolates examined in the present study, seven were S. scabies and three were S. europaeiscabiei. Although S. scabies, S. europaeiscabiei, and S. stelliscabiei were found on both continents, based on the limited results from this study and from the French study, the relative proportions of each seem to vary. The PCR primer sets developed in this study can be used to characterize larger populations of scab-causing streptomycetes from various regions to establish whether there are regional differences.

Fig. 1. (continued from preceding page)

\section{5 variable region

$\begin{array}{ll}\text { scab2m } & \\ 971124 & \text { scabiei } \\ \text { AJ007423 } & \text { europaeiscabiei } \\ \text { T2St2 } & \\ 5263074 & \text { stelliscabiei } \\ \text { turg2m } & \\ \text { AB026221 } & \text { turgidiscabies } \\ \text { acidi2 } & \\ \text { D63868 } & \text { bottropensis } \\ 971132 & \text { diastatochromog } \\ \text { AB026220 acidiscabies } & \\ \text { ID01-6.2A } & \text { group X }\end{array}$

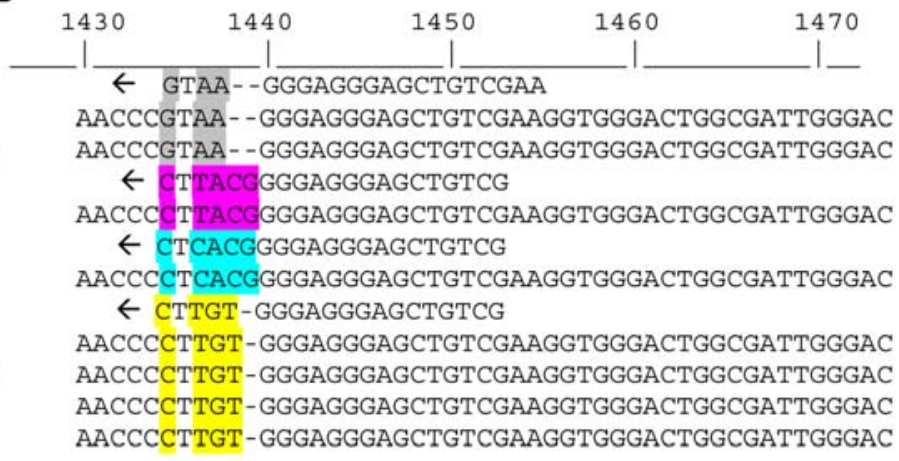

OH01-5D

OH01-6A

OH01-6.2A

OH01-12C

NY02-1B

ME01-11h

ME01-17h

MI02-2

ID01-16C

ID01-6.2A

ID01-12C

NY02 - $1 A$

NYO2 - 3A
AACCCGTAAA--GGGAGGGAGCTGTCGAAGGTGGGACTGGCGATTGGGAC AACCCGTAA - - GGGAGGGAGCTGTCGAAGGTGGGACTGGCGATTGGGAC AACCCGTAA - -GGGAGGGAGCTGTCGAAGGTGGGACTGGCGATTGGGAC AACCCGTAA - -GGGAGGGAGCTGTCGAAGGTGGGACTGGCGATTGGGAC AACCCGTAA - - GGGAGGGAGCTGTCGAAGGTGGGACTGGCGATTGGGAC AACCCGTAA - - GGGAGGGAGCTGTCGAAGGTGGGACTGGCGATTGGGAC AACCCGTAA - -GGGAGGGAGCTGTCGAAGGTGGGACTGGCGATTGGGAC AACCCGTAA - -GGGAGGGAGCTGTCGAAGGTGGGACTGGCGATTGGGAC AACCCGTAA - - GGGAGGGAGCTGTCGAAGGTGGGACTGGCGATTGGGAC AACCCCTTGT-GGGAGGGAGCTGTCGAAGGTGGGACTGGCGATTGGGAC AACCCCTTGT - GGGAGGGAGCTGTCGAAGGTGGGACTGGCGATTGGGAC AACCCCT TACGGGGAGGGAGCTGTCGAAGGTGGGACTGGCGATTGGGAC AACCCCTTACGGGGAGGGAGCTGTCGAAGGTGGGACTGGCGATTGGGAC 


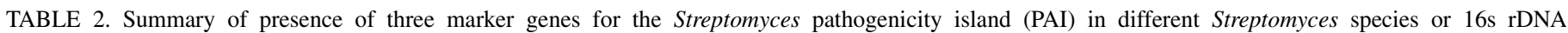
backgrounds in U.S. isolate collection

\begin{tabular}{|c|c|c|c|c|c|c|c|c|c|c|c|c|}
\hline \multirow[b]{2}{*}{ rDNA type } & \multicolumn{3}{|c|}{ Features of PAI } & \multirow{2}{*}{\multicolumn{2}{|c|}{ Pathogenicity $^{\mathrm{d}}$}} & \multirow[b]{2}{*}{ ID } & \multirow[b]{2}{*}{ WI } & \multirow[b]{2}{*}{ MI } & \multirow[b]{2}{*}{$\mathrm{OH}$} & \multirow[b]{2}{*}{ NY } & \multirow[b]{2}{*}{ ME } & \multirow[b]{2}{*}{ Total } \\
\hline & $t x t A B^{\mathrm{a}}$ & $n e c 1^{\mathrm{b}}$ & $\operatorname{tom} A^{\mathrm{c}}$ & & & & & & & & & \\
\hline \multirow{5}{*}{$\begin{array}{l}\text { S. scabies/S. } \\
\text { europaeiscabiei }\end{array}$} & + & + & + & + & {$[43 ; 18]$} & 6 & 7 & 4 & 7 & 2 & 18 & 44 \\
\hline & + & - & + & + & {$[1 ; 0]$} & 0 & 1 & 0 & 0 & 0 & 0 & 1 \\
\hline & + & - & - & + & {$[2 ; 2]$} & 1 & 0 & 1 & 0 & 0 & 0 & 2 \\
\hline & - & + & + & - & {$[1 ; 0]$} & 0 & 1 & 0 & 0 & 0 & 0 & 1 \\
\hline & - & - & - & $(-)$ & {$[0 ; 0]$} & 1 & 0 & 0 & 0 & 0 & 0 & 1 \\
\hline \multirow[t]{2}{*}{ S. stelliscabiei } & + & + & + & + & {$[7 ; 1]$} & 1 & 0 & 0 & 0 & 6 & 0 & 7 \\
\hline & - & - & - & $(-)$ & & 0 & 0 & 0 & 0 & 0 & 0 & 0 \\
\hline \multirow[t]{3}{*}{$\mathrm{X}$} & + & + & + & $(+)$ & & 0 & 0 & 0 & 0 & 0 & 0 & 0 \\
\hline & + & - & + & + & {$[5 ; 2]$} & 5 & 0 & 0 & 0 & 0 & 0 & 5 \\
\hline & - & - & - & - & {$[3 ; 1]$} & 1 & 2 & 0 & 0 & 0 & 0 & 3 \\
\hline \multirow[t]{2}{*}{ S. acidiscabies } & + & + & + & $(+)$ & & 0 & 0 & 0 & 0 & 0 & 0 & 0 \\
\hline & - & - & - & - & {$[2 ; 1]$} & 0 & 1 & 0 & 0 & 0 & 1 & 2 \\
\hline \multirow[t]{2}{*}{ S. bottropensis } & + & + & + & $(+)$ & & 0 & 0 & 0 & 0 & 0 & 0 & 0 \\
\hline & - & - & - & - & {$[2 ; 0]$} & 2 & 0 & 0 & 0 & 0 & 1 & 3 \\
\hline \multirow{2}{*}{$\begin{array}{l}\text { S. stelliscabiei }+ \\
\text { S. bottropensis }\end{array}$} & + & + & + & $(+)$ & & 0 & 0 & 0 & 0 & 0 & 0 & 0 \\
\hline & - & - & - & - & {$[16 ; 9]$} & 9 & 1 & 0 & 0 & 0 & 7 & 17 \\
\hline \multirow[t]{3}{*}{ Not identified } & + & + & + & $(+)$ & & 0 & 0 & 0 & 0 & 0 & 0 & 0 \\
\hline & - & - & - & - & {$[9 ; 0]$} & 0 & 2 & 4 & 0 & 0 & 6 & 12 \\
\hline & - & - & + & - & {$[1 ; 0]$} & 0 & 0 & 0 & 0 & 0 & 1 & 1 \\
\hline Total & & & & & {$[92 ; 34]$} & 26 & 15 & 9 & 7 & 8 & 34 & 99 \\
\hline
\end{tabular}

a Presence or absence of the thaxtomin biosynthesis operon $t x t A B$ as determined by polymerase chain reaction (PCR).

b Presence or absence of the necl gene as determined by PCR.

c Presence or absence of the tomatinase gene tomA as determined by PCR.

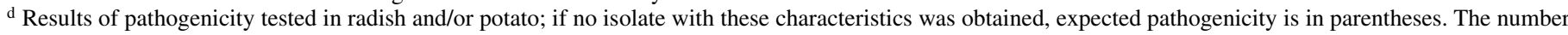
of isolates tested for pathogenicity is in brackets [number tested in radish; number tested in potato].

Only nonpathogenic representatives of $S$. acidiscabies, another species known to cause scab in the United States, Korea, and Japan $(19,33,34)$, were found in the present isolate collection. No pathogenic $S$. bottropensis was found, although this species was represented in nonpathogenic Streptomyces isolates. Although the presence of $S$. reticuliscabiei was not specifically investigated in this study, all of the isolates having the $t x t A B$ genes and pathogenic on radish or potato were classified into four Streptomyces species. This suggests that the netted scab pathogen was not a predominant species in the six regions of the United States studied, or that $S$. reticuliscabiei might lack the PAI genes analyzed.

Streptomycetes pathogenic on potato but lacking thaxtomin production (and the necl gene) have been described from Korea (28). However, pathogenicity in radish and potato corresponded to the presence of the $\operatorname{txt} A B$ genes in all 92 isolates assayed in the present study, clearly supporting the role of thaxtomin as a pathogenicity determinant in U.S. isolates of Streptomyces.

Approximately $40 \%$ of the isolates studied were nonpathogens. Nonpathogenic isolates were more diverse in morphology than pathogenic isolates and many could not be classified into the same 16 s rDNA sequence groups as pathogenic strains. Different taxonomic positions for nonpathogenic isolates from potato have been described by Doumbou et al. (7), and many nonpathogenic isolates were not identified to a specific Streptomyces species in other studies $(1,21)$.

A few isolates have an atypical composition of PAI marker genes. An isolate from Idaho and one from Michigan have $t x t A B$ and are pathogens, but lacked both necl and tomA loci located at the other end of the PAI in S. turgidiscabies, some $300 \mathrm{~kb}$ from txtAB. One Wisconsin strain has both tomA and necl, but lacks $\operatorname{txt} A B$ as determined by the PCR assay used here. Although homologous copies of these genes altered in one of the PCR primer sites would potentially be missed using the PCR assay, Southern blots of genomic DNA probed with a 700-bp fragment representing more than half of the necl gene confirm that necl homologs are missing in four isolates tested that lack necl by PCR, including two isolates that lack both tomA and necl. Patho- genic isolates missing necl have been described previously (18, 28,35). Discovery of several isolates with different subsets of the three PAI marker genes in this study suggests that variability in this region may be relatively common.

The genetic diversity found in this relatively small survey of streptomycetes isolated from field-grown potato tubers from six locations in the United States suggests that different populations of scab-causing species could contribute to differences in incidence and severity of potato common scab in different locations and years. Four pathogenic species were identified, based on sequence of variable regions in the 16s rRNA gene, and there was apparent regional variation in their distribution. Pathogenic isolates lacking one or more of three loci associated with the PAI were found, although all pathogenic isolates had genes for biosynthesis of the pathogenicity determinant thaxtomin. This report provides baseline data on the population genetics of scab-causing streptomycetes in the United States.

\section{ACKNOWLEDGMENTS}

Technical assistance by M. Elson, J. Lankasky, P. Goel, and S. Ray is gratefully acknowledged. Thanks to M. Reinsel and J. Hammond of the USDA-ARS/US National Arboretum Floral and Nursery Crops Lab for help with DNA sequencing. Scabby potato tubers were provided by J. Miller (University of Idaho Research and Extension Center, Aberdeen, ID), R. Novy (USDA-ARS, Aberdeen, ID), K. Haynes (USDA-ARS, Beltsville, MD), N. Olsen (University of Idaho Cooperative Extension Service, Twin Falls, ID), R. Slattery, Hancock, WI, E. Hollister (Michigan State University), W. Stevenson (University of Wisconsin), D. Halseth (Cornell University), and M. Martin (Potato Introduction Service, Sturgeon Bay, WI). Thanks to G. Lazarovits (Agriculture Agri-Food Canada,) and D. Labeda (USDA-ARS National Center for Agricultural Utilization Research) for very useful critical review of the manuscript.

\section{LITERATURE CITED}

1. Bouchek-Mechiche, K., Gardan, L., Normand, P., and Jouan, B. 2000. DNA relatedness among strains of Streptomyces pathogenic to potato in France: Description of three new species, S. europaeiscabiei sp. nov. and 
S. stelliscabiei sp. nov. associated with common scab, and S. reticuliscabiei sp. nov. associated with netted scab. Int. J. Syst. Evol. Microbiol. 50:91-99.

2. Bouchek-Mechiche, K., Guerin, C., Jouan, B., and Gardan, L. 1998. Streptomyces species isolated from potato scabs in France: Numerical analysis of "Biotype-100" carbon source assimilation data. Res. Microbiol. 149:653-663.

3. Bouchek-Mechiche, K., Pasco, C., Andrivon, D., and Jouan, B. 2000. Differences in host range, pathogenicity to potato cultivars and response to soil temperature among Streptomyces species causing common and netted scab in France. Plant Pathol. 49:3-10.

4. Bukhalid, R. A., Chung, S. Y., and Loria, R. 1998. nec1, a gene conferring a necrogenic phenotype, is conserved in plant-pathogenic Streptomyces spp. and linked to a transposase pseudogene. Mol. Plant-Microbe Interact. 11:960-967.

5. Bukhalid, R. A., Takeuchi, T., Labeda, D., and Loria, R. 2002. Horizontal transfer of the plant virulence gene, nec 1 , and flanking sequences among genetically distinct Streptomyces strains in the Diastatochromogenes cluster. Appl. Environ. Microbiol. 68:738-744.

6. Doering-Saad, C., Kampfer, P., Manulis, S., Kritzman, G., Schneider, J., Zakrzewska-Czerwinska, J., Schrempf, H., and Barash, I. 1992. Diversity among Streptomyces strains causing potato scab. Appl. Environ. Microbiol. 58:3932-3940.

7. Doumbou, C. L., Akimov, V., Cote, M., Charest, P. M., and Beaulieu, C. 2001. Taxonomic study on nonpathogenic streptomycetes isolated from common scab lesions on potato tubers. Syst. Appl. Microbiol. 24:451456.

8. Faucher, E., Otrysko, B., Paradis, E., Hodge, N. C., Stall, R. E., and Beaulieu, C. 1993. Characterization of streptomycetes causing Russett scab in Quebec. Plant Dis. 77:1217-1220.

9. Faucher, E., Savard, T., and Beaulieu, C. 1992. Characterization of actinomycetes isolated from common scab lesions on potato tubers. Can. J. Plant Pathol. 14:197-202.

10. Goyer, C., Faucher, E., and Beaulieu, C. 1996. Streptomyces caviscabies sp. nov., from deep-pitted lesions in potatoes in Québec, Canada. Int. J. Syst. Bacteriol. 46:635-639.

11. Haynes, K. G., Goth, R. W., and Young, R. J. 1997. Genotype X environment interactions for resistance to common scab in tetraploid potato. Crop Sci. 37:1163-1167.

12. Haynes, K. G, Wanner, L. A., Thill, C. A., Novy, R. G., and Whitworth, J. L. 2006. Common scab trials of potato varieties and advanced selections in 2003. Am. Potato J. Res. 83:113.

13. Healy, F. G., Bukhalid, R. A., and Loria, R. 1999. Characterization of an insertion sequence element associated with genetically diverse plant pathogenic Streptomyces spp. J. Bacteriol. 181:1562-1568.

14. Healy, F. G., and Lambert, D. H. 1991. Relationships among Streptomyces spp. causing potato scab. Int. J. Syst. Bacteriol. 41:479-482.

15. Hill, J., and Lazarovits, G. 2005. A mail survey of growers to estimate potato common scab prevalence and economic loss in Canada. Can. J. Plant Pathol. 27:46-52.

16. Hiltunen, L. H., Weckman, A., Ylhäinen, A., Rita, H., Richter, E., and Valkonen, J. P. T. 2005. Responses of potato cultivars to the common scab pathogens, Streptomyces scabies and S. turgidiscabies. Ann. Appl. Biol. 146:395-403.

17. Kers, J., Cameron, K., Joshi, M., Bukhalid, R., Morello, J., Wach, M., Gibson, D., and Loria, R. 2005. A large, mobile pathogenicity island confers plant pathogenicity on Streptomyces species. Mol. Microbiol. 55:1025-1033.

18. Kreuze, J. F., Suomalainen, S., Paulin, L., and Valkonen, J. P. T. 1999.
Phylogenetic analysis of 16S rRNA genes and PCR analysis of the nec1 gene from Streptomyces spp. causing common scab, pitted scab, and netted scab in Finland. Phytopathology 89:462-469.

19. Lambert, D. H., and Loria, R. 1989. Streptomyces acidiscabies sp. nov. Int. J. Syst. Bacteriol. 39:393-396.

20. Lapwood, D. H., Wellings, L. W., and Hawkins, J. H. 1973. Irrigation as a practical means to control potato common scab (Streptomyces scabies): Final experiment and conclusions. Plant Pathol. 22:35-41.

21. Lehtonen, M. J., Rantala, H., Kreuze, J. F., Bang, H., Kuisma, L., Koski, P., Virtanen, E., Vihlman, K., and Valkonen, J. P. T. 2004. Occurrence and survival of potato scab pathogens (Streptomyces species) on tuber lesions: Quick diagnosis based on a PCR-based assay. Plant Pathol. 53:280-287.

22. Levick, D. R., Evans, T. A., Stephens, C. T., and Lacy, M. L. 1985. Etiology of radish scab and its control through irrigation. Phytopathology 75:568-572.

23. Lindholm, P., Kortemaa, H., Haahtela, K., Kokkola, M., SalkinojaSalonen, M., and Valkonen, J. P. T. 1997. Streptomyces spp. isolated from potato scab lesions under nordic conditions in Finland. Plant Dis. 81:1317-1322.

24. Loria, R. 2001. Diseases caused by bacteria: Common scab. Pages 14-15 in: Compendium of Potato Diseases. 2nd ed. W. R. Stevenson, R. Loria, G. D. Franc, and D. P Weingartner, eds. The American Phytopathological Society, St. Paul, MN.

25. Loria, R., Bukhalid, R. A., Fry, B. A., and King, R. R. 1997. Plant pathogenicity in the genus Streptomyces. Plant Dis. 81:836-846.

26. Miyajima, K., Tanaka, F., Takeuchi, T., and Kuninaga, S. 1998. Streptomyces turgidiscabies sp. nov. Int. J. Syst. Bacteriol. 48:495-502.

27. Park, D. H., Kim, J. S., Kwon, S. W., Wilson, C., Yu, Y. M., Hur, J. H., and Lim, C. K. 2003. Streptomyces luridiscabiei sp. nov., Streptomyces puniciscabiei sp. nov. and Streptomyces niveiscabiei sp. nov., which cause potato common scab disease in Korea. Int. J. Syst. Evol. Microbiol. 53:2049-2054.

28. Park, D. H., Yu, Y. M., Kim, J. S., Cho, J. M., Hur, J. H., and Lim, C. K. 2003. Characterization of streptomycetes causing potato common scab in Korea. Plant Dis. 87:1290-1296.

29. Pasco, C., Jouan, B., and Andrivon, D. 2005. Resistance of potato genotypes to common and netted scab-causing species of Streptomyces. Plant Pathol. 54:383-392.

30. Powelson, M. L., Johnson, K. B., and Rowe, R. C. 1993. Management of diseases caused by soilborne pathogens. Pages 154-156 in: Potato Health Management. R. C. Rowe, ed. The American Phytopathological Society, St. Paul, MN.

31. Sambrook, J., Fritsch, F., and Maniatis, T. (eds.) 1982. Molecular Cloning: A Laboratory Manual. 3rd ed. Cold Spring Harbor Laboratory, Cold Spring Harbor, NY.

32. Schaad, N. W., Jones, J. B., and Chun, W. (eds.) 2001. Laboratory Guide for the Identification of Plant Pathogenic Bacteria. The American Phytopathological Society, St. Paul, MN.

33. Song, J., Lee, S.-C., Kang, J.-W., Baek, H.-J., and Suh, J.-W. 2004. Phylogenetic analysis of Streptomyces spp. isolated from potato scab lesions in Korea on the basis of 16S rRNA gene and 16S-23S rDNA internally transcribed spacer sequences. Int. J. Syst. Evol. Microbiol. 54:203-209.

34. Tóth, L., Maeda, M., Tanaka, F., and Kobayashi, K. 2001. Isolation and identification of pathogenic strains of Streptomyces acidiscabies from netted scab lesions of potato tubers in Hokkaido (Japan). Acta Microbiol. Immuno. Hung. 48:575-585.

35. Wanner, L. A. 2004. Field isolates of Streptomyces differ in pathogenicity and virulence on radish. Plant Dis. 88:785-796. 